\title{
ACTIVE NETWORKS NODES FOR ADAPTIVE MULTIMEDIA COMMUNICATION
}

\author{
Otto Spaniol, Jens Meggers \\ RWTH Aachen, Department of Computer Science 4 \\ Ahornstr. 55, 52056 Aachen, Germany
}

\begin{abstract}
This paper proposes the use of active networking technology for congestion control and fast error correction with application level error recovery code deployed in active nodes. Its focus lies on real-time video transmission of sender based adaptive applications which are supported by active network nodes. Experiments with a prototype model show a considerable improvement with respect to video quality, error correction time, and bandwidth utilisation
\end{abstract}

\section{INTRODUCTION}

Active networks introduce a new powerful communication paradigm that provides substantial benefits for multimedia communication: nodes within the network become able to execute application or user specific code that may introduce new protocol elements or process incoming data streams. This enables network nodes to adapt traffic by application specific methods which are installed in network nodes, or even are part of the transported packet streams. This promising approach has a great deal of unexplored potential: applications are freed from complex processing and new highlevel protocols are dynamically integrated without changing network software. Furthermore, new user specific adaptive algorithms become possible which increase the flexibility of multimedia communication systems.

A highly flexible multimedia communication system is of great importance when considering the requirements of upcoming applications, services, and heterogeneous networks. Multimedia communication requires 
high bandwidth, low packet delay, low jitter, and also small packet loss rates. In particular, mobile multimedia communication demands flexible resource management and highly adaptive data streams that are able to adapt to varying packet error rates and sudden network congestion caused, e.g., by terminal mobility.

In addition to applications like network management or dynamic protocol deployment, two still existing problems in multimedia communication can be addressed by means of active network nodes: congestion control at network bottlenecks and application level error recovery. Dedicated application-specific procedures within a set of network nodes enable streamspecific traffic shaping. Instead of adapting the transmission throughput at the transmitting application, congestion can better be directly controlled at the network bottleneck, leading to fast bandwidth adaptation and minimal packet loss. Especially in heterogeneous networks, bottlenecks arise at gateway routers that connect different network technologies, e.g. wireless and fixed networks. Here, congestion control by active network nodes can contribute to improved interactive real-time multimedia communication.

This paper describes how active network nodes can support both congestion and error control for real-time video streams. We show how sender based adaptive applications can benefit from active nodes and describe a traffic reduction and error control scheme that substantially increases the overall end-to-end video communication quality.

\section{CONGESTION CONTROL}

Interactive real-time multimedia streams have strong requirements concerning packet loss, end-to-end delay, and jitter. As the Internet does not support these type of applications, different solutions have been proposed that promise the reduction of network congestion and consequently reduce packet loss and end-to-end delay.

\section{Sender Adaptation}

Sender based adaptation requires adaptive applications [DHT95] that adapt their output rate to the present network condition. The Direct Adjustment Algorithm [Sis97] for example decreases the senders' data rate when detecting network congestion. In case of a non-congested state, the sender increases its transmission speed periodically until a congestion is detected. The scheme is designed analogous to the TCP flow control scheme. Some schemes claim to be TCP-friendly [RHE99], which means that the available bandwidth is shared between TCP and the adaptive multimedia data streams without letting TCP streams dominate the others, or vice versa. The main disadvantage of the adaptive sender approach is the fact that the sender is only notified after the reception quality has been 
deteriorated. Thus, redundant coding becomes necessary that allows to compensate for a certain amount of packet loss. Another drawback is that the sender would have to adapt its transmission rate to the available bandwidth of the weakest receiver when multicast transmission is used. In scenarios with a high number of participants this would lower the overall delivery quality even for users connected by a reliable high speed link.

\section{Receiver Adaptation}

The receiver based adaptation approach [MJV96] has been designed to solve the multicast disadvantage of the sender based adaptation scheme. Here, the receiver simulcasts separate streams of different quality layers of the transmitted stream. The receiver detects packet losses and subscribes or un-subscribes to certain layers in order to meet a specific throughput. The receiver based approach has shown to perform well in multicast scenarios but is limited by the number and granularity of the separated sub-streams. The multimedia content needs to be very flexible in order to allow the splitting into many different sub-streams. Although latest video compression techniques like H.263+ [Côt98] enable multi-layered coding, the encoding process becomes more complex and produces more overhead.

\section{Resource Reservation}

When using resource reservation, applications signal their delay and throughput requirements to the appropriate network nodes and receive an admission control acknowledgement [FRV92] [CSZ92]. In case of a negative acknowledgement they have to reduce the desired resources and start a new request. To guarantee a certain delay or packet loss rate, applications would have to reserve their peak data rate, leading to poor network utilisation. Sophisticated measurement based algorithms [JDS95] try to increase the utilisation by allowing mean rate allocation. However, these measurement based schemes only work at a high amount of statistical multiplexing that is not given in some networks, e.g. mobile networks. Another disadvantage is that even measurement based approaches do not reach a utilisation above $80 \%$ and cannot avoid packet losses or delay violations. A further practical disadvantage of resource reservation is its impracticability in wireless networks where end-systems move and the error characteristic of the wireless transmission medium may change over time. The network's resource reservation would have to track the movement of the mobile terminal, leading to unpredictable resource availability. Although some schemes exist that allow admission control for mobile users [TAL97], the call blocking probability increases drastically with rising network utilisation.

\section{Priority Systems}

Priority systems such as type of service routing or the differentiated services approach [Bloa98] allow for service differentiation and enable the deployment of packet schedulers that give real-time packets priority over 
other service classes [FlJ95]. Despite the usefulness of such systems, the congestion problem is still present within a single service class.

Active Networks

A solution that makes use of active network nodes is to adapt the upcoming traffic directly at the congested node. This can be done by installing dedicated procedures that are able to reduce the required bandwidth in case of a congestion within a network node. The required adaptation procedures can be transported by the multimedia stream itself in order to allow stream specific adaptation. A simple active network could have fixed, pre-installed adaptation procedures that are selected according to the payload type or the congestion state. The active network solution is very interesting because it works well in multicast systems, allows for high bandwidth utilisation, and can avoid packet loss and delay violation. However, such a system comes at a cost: a complex network infrastructure is needed; additional security problems arise; new applications become necessary and the active nodes have to consider an additional spare resource - CPU time. Nevertheless, there are many application where the advantage of such a solution outweigh the disadvantages. For example, a wireless cellular network consists of many small cells, each serving only few users. Here, the additional required CPU resources would not be too high and would allow to avoid packet loss and delay violations.

In order to allow for fair link sharing and limited CPU usage at the active node, bandwidth adaptation by means of active network nodes can be combined with the above mentioned congestion schemes. For example, a resource reservation system can make use of an active network in order to implement dynamic traffic filters [WiZ98], or a differentiated service system can deploy active network elements for traffic shaping and metering.

Network support for applications that make use of end-to-end flow control and sender based adaptation schemes is another promising approach. Here, active nodes could be placed at selected network bottlenecks to avoid a decreasing application level communication quality due to packet loss. In the following, this approach is examined in more detail.

\section{THE ADAPTIVE ACTIVE NETWORK APPROACH}

The approach presented in this paper is to install active nodes at network bottlenecks to increase the application level quality of service of adaptive applications. An adaptive application that transmits video streams controls its output rate by adjusting the video picture quality according to the filling grade of the transmitters' output queue (local feedback) [DHT95]. Receivers 
periodically send packet loss reports to the sender application that calculates a maximum output rate and configures its transmitter appropriately (receiver feedback).

Figure 3.1 shows the combination of an active network node with an adaptive application. By installing an active network node in the transport path from the transmitter to the receiver, short term network congestion control and error recovery can be done without affecting the participating applications. The active node can predict congestion by monitoring the number of packets that reside in its input or output buffers (congestion prediction). When necessary, the active node starts application specific procedures that reduce the amount of data per stream.

We propose to install active network nodes at network routers which form bottlenecks. These routers connect different network types, e.g. a core network of a cellular network to the Internet.

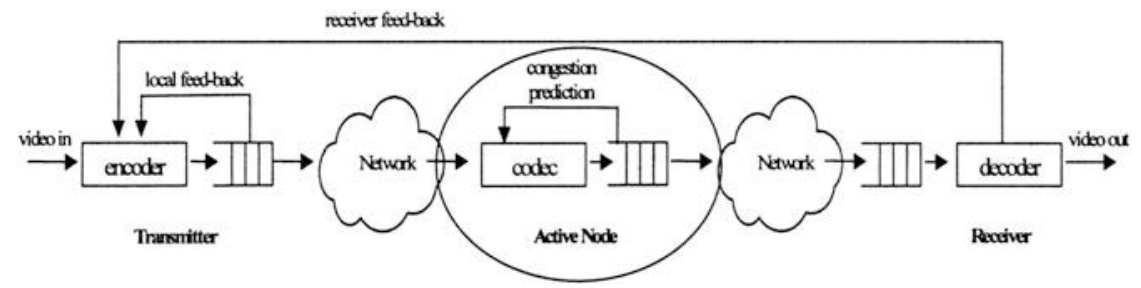

Figure 3.1. Deploying Adaptive Applications with Active Nodes

An active network has many advantages that help to install and initiate application specific adaptation procedures within the network. It allows to install transcoder units that may (dynamically) reduce the amount of bandwidth consumed by any given data stream. As these procedures depend on the respective application, active networks provide a flexible framework enabling the installation and initiation of user specific adaptation procedures within the network. In addition, active network nodes may initiate error recovery processes that are specific to the transported streams. For example, a multicast delivery of a newspaper would require packet re-transmission in case of a packet loss, while a real-time transmission of a video stream would require to update the video frame content of the following frame.

In the following section a sample implementation of an active node that operates on H.261 video streams is shown. Although the described prototype implementation processes H.261 video streams, the results should be viewed independent of this coding technology. Similar results can be achieved with other encoding schemes and algorithms. It is more important to understand that the active node's process depends on the application and the user preferences. Consequently, it is of great importance that the active node can 
be (dynamically) configured to support other adaptation processes depending on user and application requirements.

\section{CONGESTION CONTROL}

In order to investigate congestion control by active network nodes, we prototyped a sender based adaptive application and an active node. The sender transmits intra coded H.261 [H261] video streams with a conditional replenishment algorithm via RTP [Sch96] as implemented in the vic and IVS system [MCC95][TUR96]. End-to-end bandwidth adaptation is done by the Direct Adjustment Algorithm (DAA) [Sis97]. The DAA algorithm increases the output rate by an Additive Increase Factor $(A I F)$ when the reported loss remains below a certain loss threshold. When the loss rate is reported above a pre-defined threshold, the output rate is reduced by the loss-rate reported from the receiver.

The transmitter configures its throughput to the value estimated by the DAA scheme by changing the quantisation parameter of the H.261 encoder [BoT94]. Because the resulting output bandwidth is of variable bitrate, it is difficult for a sender to exactly meet an envisaged output rate. Thus, only the mean output rate of the video transmitter is controlled, sometimes leading to an output rate above the calculated value. According to our measurements this may cause unfairness. We modified the DAA scheme to calculate the new output bitrate from the actually measured rate if the measured output rate is higher than the envisaged output rate. This modification ensures that a source transmitting at a rate higher than the one given by the DAA scheme is reduced by a fraction of the actual transmitted data. The modified DAA scheme is as follows:

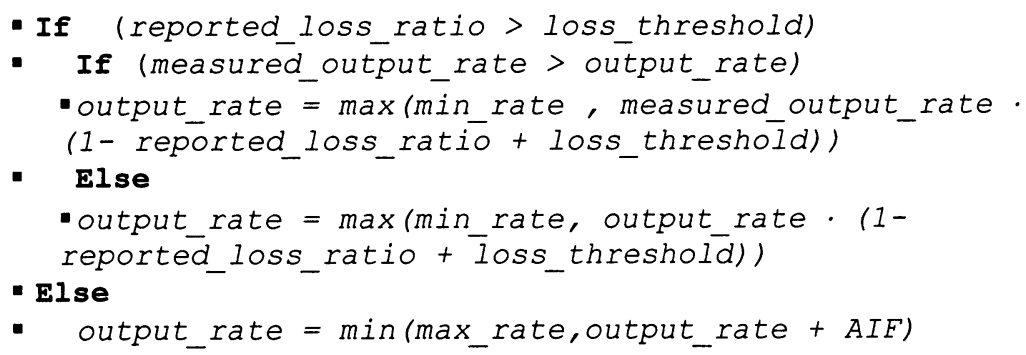

We implemented the active node as an RTP translator [Sch96] with a single output queue. The packets are serviced by a FCFS scheduling discipline. Figure 4.1 shows the experimental set-up. Four video transmitters send video streams through the active node to a corresponding destination. 
The transmitters are interconnected by a $100 \mathrm{Mbit} / \mathrm{s}$ Fast Ethernet and the output of the active node is configured to transmit with a maximum output rate of $1,5 \mathrm{Mbit} / \mathrm{s}$. Thus, the four sources will have to share $1.5 \mathrm{Mbit} / \mathrm{s}$. The minimum transmission rate of each transmitter is set to $228 \mathrm{kbit} / \mathrm{s}$ (min_rate) and the maximum to $1,5 \mathrm{Mbit} / \mathrm{s}$ (max_rate). The AIF is configured to 50 $\mathrm{kbit} / \mathrm{s}$. The loss threshold is set to $5 \%$.

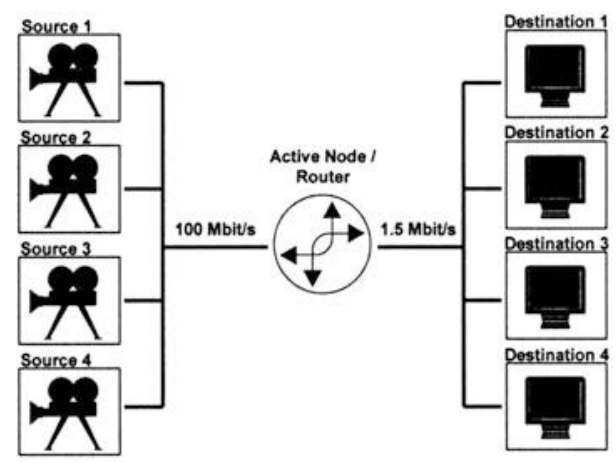

Figure 4.1. Experiment Configuration

Figure 4.2 shows how the bandwidth is shared by the 4 video sources. Every 3 minutes, another source is started. It can be seen that the bandwidth is equally shared and that newly incoming streams force the other sources to reduce their bandwidth usage.

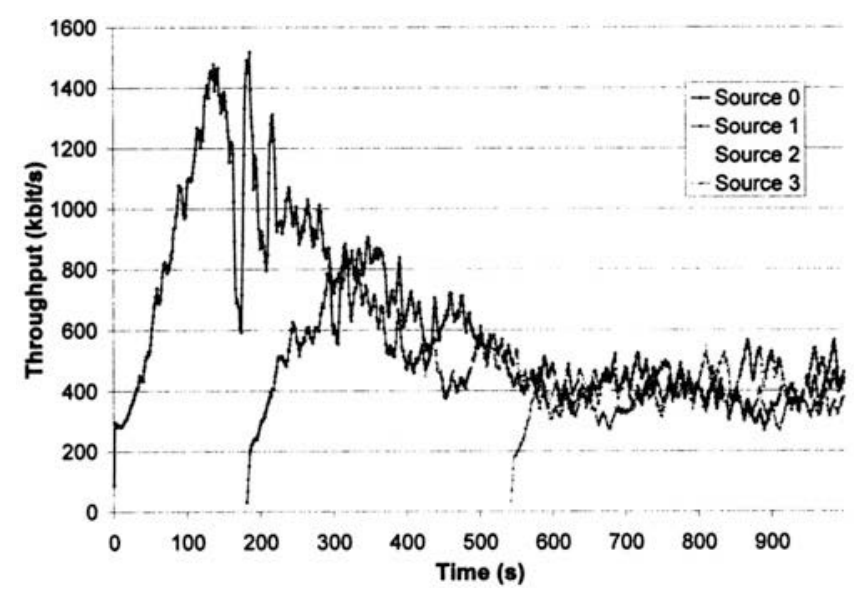

Figure 4.2. Bandwidth share controlled by the DAA scheme 
We have implemented a simple bandwidth reduction scheme at the active node that does H.261 packet by packet transcoding. Although we transcode the incoming stream from H.261 to H.261, we have chosen the transcoding approach in order to be more flexible in adapting bandwidth, e.g. by changing the frame-rate, video colours, or by a picture size reduction.

We have chosen the DAA for end-to-end bandwidth adaptation in order to relieve the active node from instantly reducing the throughput. When all participating sources transmit with a constant bit rate, the estimated throughput of $n$ sources can exceed the maximum available bandwidth by $n$. $A I F$ plus the loss threshold fraction of the maximum available bandwidth. Thus, the active node would only have to reduce the amount of bandwidth used per stream by the $A I F$ and a threshold fraction of its actual rate. However, this calculation does not hold for sources with variable bitrate where bandwidth peaks may overlap. Thus, we have configured the reduction process of the active node by multiplying the prevailing quantisation of each connection by three when the output queue length of the active node exceeds $1 / 3$ of its maximum size (the maximum size was set to 100 packets). This reduced the data rate of the processed connection by more than the AIF, but on the other hand it limits the execution time for the transcoding process.

An important implementation detail of the active node is the additional delay due to packet processing. In our implementation, re-quantisation is done by transcoding on a per packet basis. This adds an additional worst case end-to-end delay of one packet decoding and encoding time, plus one packet inter-arrival time in case of packet re-segmentation. The packet decoding and encoding duration depends on the amount of macro blocks that are stored in a packet, which in turn depends on the encoder's quantisation parameter. As shown in table 4.1, the decoding duration varies between 0.13 and $0.94 \mathrm{~ms}$ and the encoding duration between 0.15 and $5.2 \mathrm{~ms}$. The encoding and decoding duration becomes significantly higher when the DCT/IDCT is processed. However, when processing DCT/IDCT, the transcoding process is not limited to a simple re-quantisation task. Instead, it would be possible to implement colour or size reduction of the video pictures. 


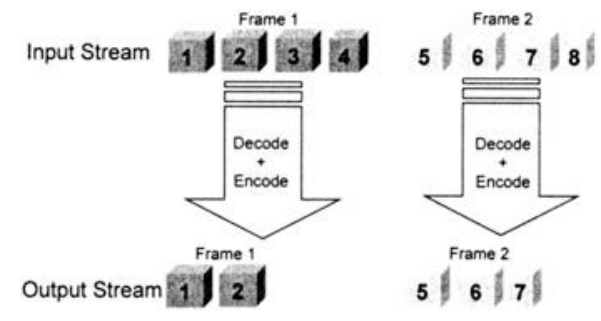

Figure 4.3. Packet by Packet Transcoding/Re-quantisation

Our implementation re-segments packets in order to reach packets sizes equal to the maximum transfer unit (MTU), to achieve the maximum performance on the destination network, and to limit packet overhead. Packets that are reduced by the re-quantisation process will be detected as packet loss at the receiver, because the active node resynchronises the RTP sequence number with the next arriving video frame. This allows end-to-end schemes like the DAA to work in conjunction with the active node. Figure 4.3 shows the packet by packet transcoding scheme.

In order to evaluate the improvement of our system, we were interested in application level quality metrics. We selected the Peak Signal to Noise Ration (PSNR) value and the video frame inter arrival time at the receiver for comparing the active node operation with a simple FIFO packet scheduler. Both versions deploy the DAA scheme on an end-to-end basis.

Table 4.1. Decoding and encoding duration for a single packet

\begin{tabular}{|c|c|c|c|c|c|}
\hline Quant & $\begin{array}{c}\text { Blocks per } \\
\text { Packet }\end{array}$ & $\begin{array}{c}\text { Decoding } \\
\text { IDCT }\end{array}$ & $\begin{array}{c}\text { Decoding } \\
\text { no IDCT }\end{array}$ & $\begin{array}{c}\text { Encoding } \\
\text { DCT }\end{array}$ & $\begin{array}{c}\text { Encoding } \\
\text { no DCT }\end{array}$ \\
\hline 30 & 66.7 & $0.94 \mathrm{~ms}$ & $0.7 \mathrm{~ms}$ & $5.2 \mathrm{~ms}$ & $0.95 \mathrm{~ms}$ \\
15 & 44.1 & $0.82 \mathrm{~ms}$ & $0.4 \mathrm{~ms}$ & $3.7 \mathrm{~ms}$ & $0.65 \mathrm{~ms}$ \\
1 & 4.5 & $0.24 \mathrm{~ms}$ & $0.13 \mathrm{~ms}$ & $0.47 \mathrm{~ms}$ & $0.15 \mathrm{~ms}$ \\
\hline
\end{tabular}

To measure the frame inter arrival deviation at the receiver, we have measured the time between two consecutive video frames at the sender and the corresponding inter arrival time at the receiver. The difference of this value for frame $i$ gives us the video frame jitter $\Delta j_{i}$. Figure 4.4 shows a histogram of $\Delta j_{i}$ measured on the basis of 10000 delivered frames. The experiments have shown that the video frame inter arrival time of the active node operation shows smaller jitter values than the simple system with a FIFO packet queue. The mean $\Delta j_{i}$ for the DAA with FIFO queuing is 32 $\mathrm{ms}$, and the average jitter for the system with active network support is 24 ms. Thus, the receiving application needs to maintain a smaller jitter buffer that directly reflects the end-to-end communication delay. The lower video frame inter arrival times are caused by smaller queuing delays in the active 
node queue because the overall packet delay is reduced by the lower average filling grade of the output queue of the active node. Another important factor is that the active node operation avoids packet loss. Instead, packets are reduced by transforming the video data to a lower quality level. Because of the segmentation of video frames on RTP packets, as described in RFC 2032, the receiving application will have to wait for the last packet belonging to the actual frame before displaying the video frame. When the last packet of a video frame is lost, the receiver cannot display the frame until a packet of the next frame arrives'.

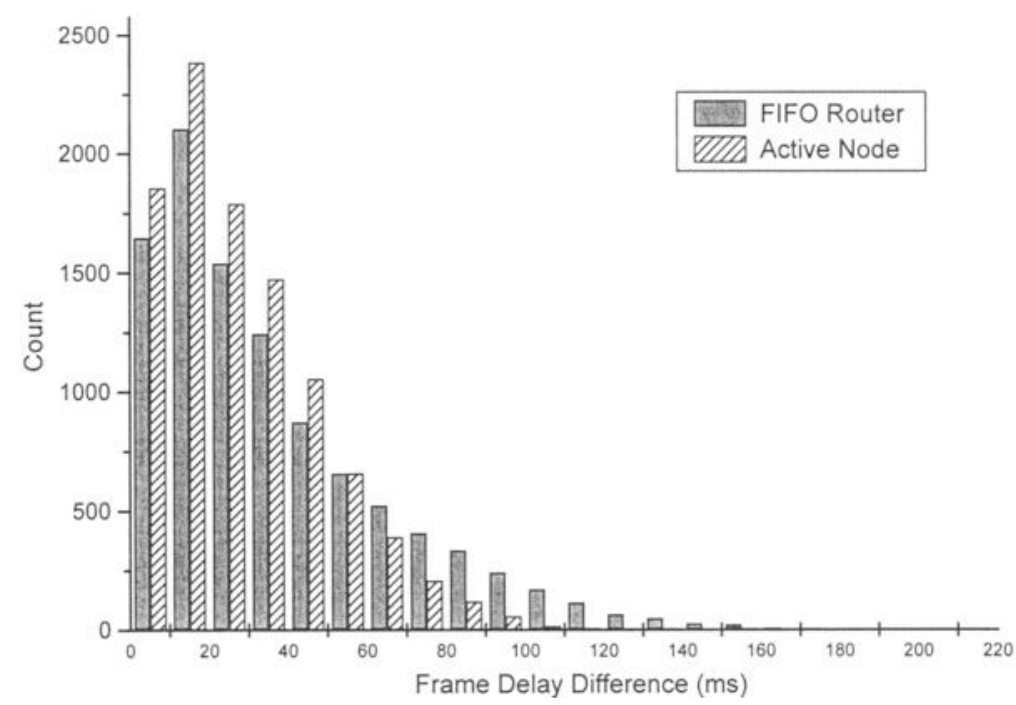

Figure 4.4. FIFO vs. Active Node Video Frame Interarrival Times

A second quality metric measured on application level is the PSNR of the video at the receiving application. Two processes affect the actual video quality. One is the sender application that configures the video encoder according to the aspired output rate. The other is the active node that lowers the video quality when the transcoding process is started.

Our prototype implementation measures the PSNR both at the receiver and the sender application simply by comparing the transmitted and received stream with the original video signal that has been stored to a file. Thus, the $\triangle \mathrm{PSNR}_{i}$ for the transmitted video frame $i$, which is a measure for the degradation of video quality during transmission, can be calculated as:

\footnotetext{
${ }^{1}$ This kind of implementation assumes that packet reordering does not take place.
} 


$$
\Delta \text { PSNR }_{i}=20 \log _{10} \frac{255 \cdot N^{2}}{\sqrt{\sum(T(i, j)-F(i, j))^{2}}}-20 \log _{10} \frac{255 \cdot N^{2}}{\sqrt{\sum(R(i, j)-F(i, j))^{2}}}
$$

where $F(i, j)$ is the input signal at the sender's encoder, $T(i, j)$ the output signal from the encoder, and $R(i, j)$ the signal received at the receiver application. $R(i, j)$ differs from $T(i, j)$ when the active node performs bandwidth adaptation or when packets are lost.

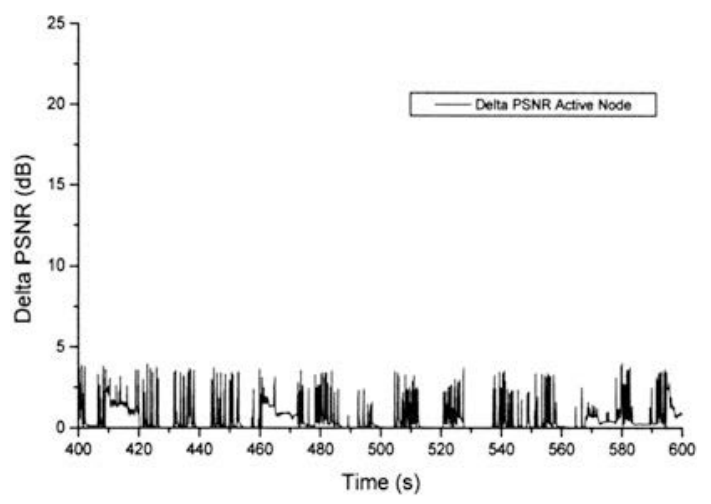

Figure 4.5 a. $\triangle \mathrm{PSNR}$ with the Active Node

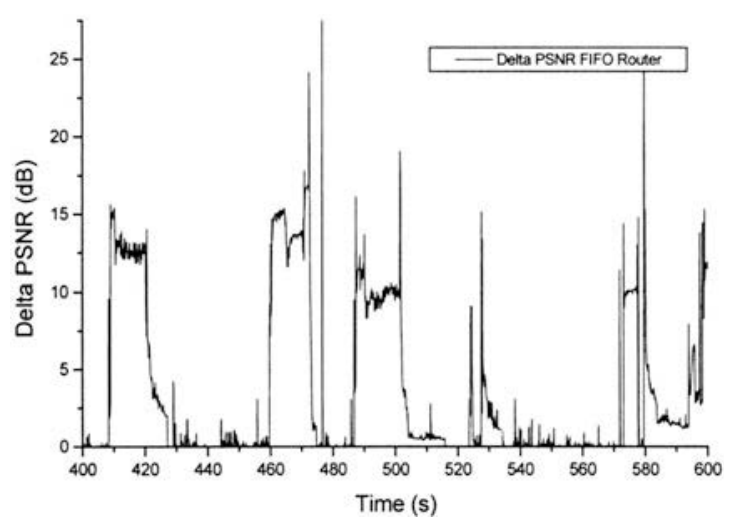

Figure 4.5 b. $\triangle \mathrm{PSNR}$ with the FIFO Router

Due to the fact that the active node reduces the video quality in case of congestion, the receiving video quality is lower than the quality at the 
sender. However, without the active node operation, packets are dropped when the queue reaches its maximum capacity. Thus, the quality without the active node is much lower than with the active node operation. Figure $4.5 \mathrm{~b}$ shows the $\triangle$ PSNR measured at the sending and receiving application when the DAA and a FIFO queue is used. Here, the mean $\triangle$ PSNR is $1.98 \mathrm{~dB}$ with a the standard deviation of $3.88 \mathrm{~dB}$.

In contrast, the active node allows for a lower $\triangle P S N R$ as depicted in Figure $4.5 \mathrm{a}$. Here, the average $\triangle \mathrm{PSNR}$ is about $0.48 \mathrm{~dB}$ with a standard deviation of $0.84 \mathrm{~dB}$. Figure $4.5 \mathrm{a}$ shows that the active node achieves a considerable improvement in quality.

\section{ERROR CONTROL}

The reason for the high $\triangle$ PSNR values of the DAA scheme without active node adaptation is as follows: when a packet from a video stream is lost, the corresponding display area will be damaged until a subsequent video frame encodes the macro blocks in intra mode. When the encoder deploys motion compensation with motion vectors, the display errors will extend over time and space. With MPEG, the error will not be corrected until the next I-frame is transmitted. The ITU standards H.261 and H.263 do not dispose the repetition of a video frame encoded in full intra frame mode. In order to allow for minimal end-to-end delay, the frame sequence can be encoded in P-frames that may skip a macro block or encode it in P- or Imode. This feature reduces the overall throughput and avoids peaks that may cause congestion or delayed transmission. However, long I-frame repeating intervals make it necessary to deploy a feedback scheme that triggers the sender's encoder to correct display errors by refreshing spatial video regions in intra mode ${ }^{2}$ [MeS99].

In [BoT98] and [MeS99], such feedback schemes have been examined. The scheme proposed in [MeS99] reports the actual state of its display to the sender by mapping the timestamps of the received packets to the corresponding display region. It has been shown that this scheme introduces only very few additional bandwidth usage [MeS99].

Instead of an end-to-end error feedback control as analysed in [MeS99], we have implemented hop by hop error recovery between active nodes. When the active node receives an error report from either the receiving application or from another active node located downstream, it inserts the

${ }^{2}$ The area of blocks to be encoded in intra mode depends on the usage and the distance of motion vectors. 
contents of the lost video regions into the next video frame. Figure 5.1 shows the configuration of our with 2 active nodes.

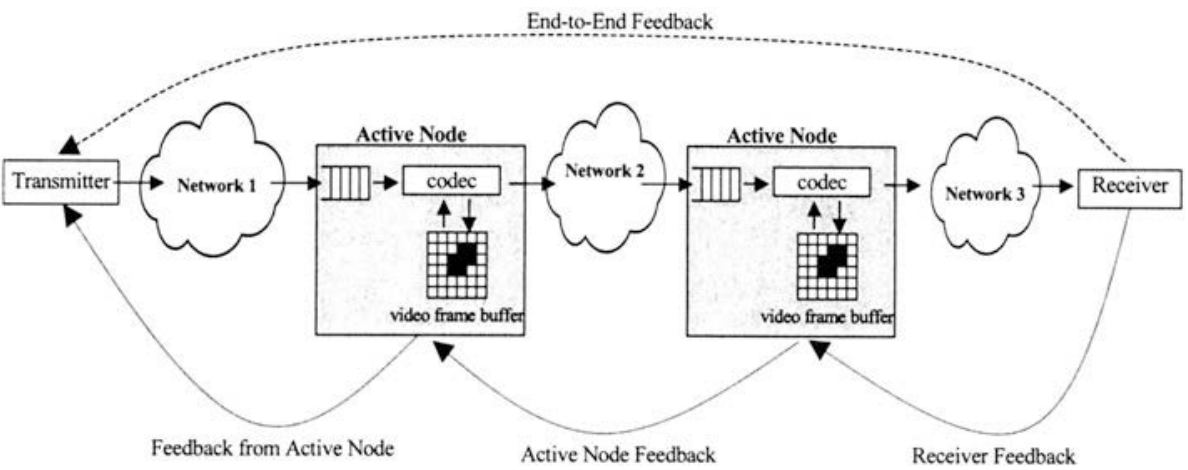

Figure 5.1. Error Control with End-To-End Feedback (Top) and Active Node Feedback (Bottom)

In order to evaluate the improvement of such a system, we installed the configuration as depicted in figure 5.1. We connected the different systems by a LAN and introduced additional queuing delay in the output queues of the transmitter and the active nodes in order to measure results comparable to a wide area network. In addition, we implemented an error simulation within the input queues of the receiver and the active nodes. The error simulation model uses a Gilbert model to simulate burst errors. This allows us to conduct experiments with different error rates. To test the behaviour of the active node error recovery within an heterogeneous network, we configured the propagation delay and the packet error rates as summarised in table 5.1. Whereas the first two networks each introduce a queuing delay of $100 \mathrm{~ms}$ and a packet error rate of $10 \%$, the last hop represents an access network a smaller propagation and queuing delay. This configuration could represent a wireless access network connected to a wired wide area network (e.g. the Internet).

Table 5.1. Experimental Queuing Delay and Packet Error Rates

\begin{tabular}{|l|c|c|c|}
\cline { 2 - 4 } \multicolumn{1}{c|}{} & \multicolumn{1}{|c|}{$\begin{array}{l}\text { Network } \\
\text { - }\end{array}$} & $\begin{array}{l}\text { Network } \\
\mathbf{2}\end{array}$ & - Network 3 \\
\hline $\begin{array}{l}\text { Packet Error } \\
\text { Rate }\end{array}$ & - $10 \%$ & - $10 \%$ & - $20 \%$ \\
\hline - Delay (ms) & - $100 \mathrm{~ms}$ & - $100 \mathrm{~ms}$ & - $20 \mathrm{~ms}$ \\
\hline
\end{tabular}

We compared the end-to-end feedback with the feedback between active nodes. After sending 4000 video frames, we observed an average $\triangle$ PSNR of $2.35 \mathrm{~dB}$ per video frame when deploying end-to-end feedback error control. 
The hop by hop feedback scheme between active nodes and applications could improve the video quality and resulted in a $\triangle$ PSNR of $1.81 \mathrm{~dB}$. Another measure for video quality is the number of macro blocks that appear on the receivers display without error. Whilst the average number of damaged macro blocks is 48,22 for the end-to-end error correction, the active nodes reduce this number by 27 percent to 35 blocks (see Table 5.2).

Table 5.2. Video Quality of the different Error recovery Configurations

\begin{tabular}{|l|c|l|}
\cline { 2 - 3 } \multicolumn{1}{c|}{} & Average $\triangle$ PSNR & $\begin{array}{l}\text { Damaged Macro Blocks per } \\
\text { Frame }\end{array}$ \\
\hline $\begin{array}{l}\text { No Error } \\
\text { Correction }\end{array}$ & 11,72 & 143,25 \\
\hline $\begin{array}{l}\text { End To End Error } \\
\text { Correction }\end{array}$ & 2,35 & 48,22 \\
\hline $\begin{array}{l}\text { Active Node Error } \\
\text { Correction }\end{array}$ & 1,81 & 35,01 \\
\hline
\end{tabular}

Figure 5.2 shows the load generated on the three connecting networks by the single source. Whilst the load of the end-to-end feedback version is reduced at each node by the packet error rate, the load when deploying active nodes is reduced less due to the error correction done by the intermediate nodes. Compared to the end-to-end error correction, the active network error correction reduces the network load in network 1 and network 2 and adds a small amount of additional bandwidth within network 3 . However the total network load of the active network system is lower than that of the end-to-end feedback scheme. Despite of this lower overall bandwidth consumption the quality of the video is improved.

\section{DISCUSSION}

Despite the benefits of active network usage for congestion and error control as described in the former sections, some questions remain that have to be solved before deploying this type of network system.

One remaining problem is the CPU resources required at the active node. Although the adaptation process will only adapt the data steams during periods of congestion, the required CPU usage during these intervals increases linearly with the number of transmitted streams. For a transcoding approach as described in the former chapter this will considerably limit the number of transported streams. However, modern coding techniques such as Wavelet coding or hierarchical coding in H.263+ and MPEG-4 promise to simplify the transformation process by simply dropping packets of lower 
coding layers. Nevertheless, the active code running in the active node is a further resource that has to be admitted and charged. One approach is proposed in the SwitchWare active network model [Ale98a]. Here, the CPU cycles per user specific code can be measured and restricted.

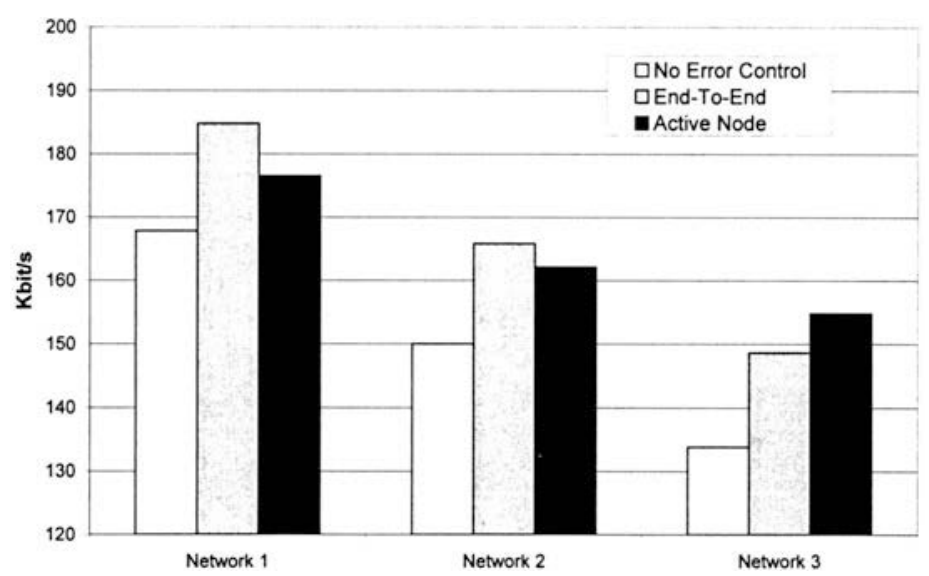

Figure 5.2. Network Load caused by the end-to-end Corrections Compared to Active Network Correction

A further argument against the deployment of active network nodes is the so called end-to-end argument, that says not to implement high level functionality within the network if it can be implemented better and more cost-effectively in end systems, especially if some applications may not benefit from such functions at all [SRC84][Bha97]. However, in [Bha97] it is shown that the end-to-end argument does not rule out high level functionality within networks. Nodes within the network may have information that is not available in end systems, such as congestion states or traffic load pattern. Thus, the combination of application level information, e.g. payload dependencies or data priority, may provide for services that can be best supported or enhanced only if application and network information are combined. Due to active networks inherent property of being application driven, and since services can be tailored to the user's requirements, the active network can be implemented cost effectively, because it is only used by applications that have configured them to their needs.

Another important consideration deals with security aspects of active networks. In addition to many security problems that arise when installing and maintaining an active network [Ale98b], it is a basic requirement from the user's point of view that the active network only allows those code segments to process the applications' data stream that have originated from the application itself. If the code has been not generated by the application, it 
has to be ensured that this code is a well known or certified one. Consequently, the active network system has to ensure that the transported or invoked code is not modified prior to or during execution. A further security problem is the active network support for encrypted data streams.

\section{CONCLUSION}

We have introduced an active network node that avoids network congestion by decreasing the video quality of transmitted video streams in periods of congestion. The active node works on the application level and thus allows to apply application specific adaptation procedures to the transported data. Our implementation makes use of a specific video transcoder to demonstrate the benefits of application level bandwidth adaptation by means of active network nodes. A sender based adaptation scheme was combined with an active network node that allows to minimise the adaptation process at the router and to perform end-to-end flow control.

We have shown that the network adaptation approach can significantly improve the end-to-end video quality, and can contribute to error correction, required when packets are dropped in network nodes that connect different active nodes.

This work has shown that application specific procedures installed within the network can significantly increase the overall service quality. Installed in a secure active network environment, application specific congestion and error control procedures may enable interactive real-time communication even in unreliable, congested networks.

\section{REFERENCES}

[Ale98a] D. S. Alexander et al, " The SwitchWare Active Network Architecture", IEEE Network Special Issue on Active and Controllable Networks, Vol. 12 No. 3, pp. 29 36, IEEE Press 1999.

[Ale99] D. S. Alexander et al, " Security in Active Networks “, Secure Internet Programming: Issues in Distributed and Mobile Object Systems, Springer-Verlag Lecture Notes in Computer Science State-of-the-Art series.

[Bha97] S. Bhattacharjee et al, "Active Networking and the End-to-End Argument", ICNP '97, Atlanta, GA, October 1997.

[Bla98] S. Blake et al, "An Architecture for Differentiated Services", RFC 2475, December 1998.

[BoT94] J. Bolot, T. Turletti, "A rate control for packet video in the Internet", Proc. IEEE INFOCOM '94, pp. 1216-1223, Toronto June 1994. 
[BoT98] J-C. Bolot, T. Turletti, "Experience with Control Mechanisms for Packet Video in the Internet", ACM SIG COMM Computer Communication Review, Vol. 28, No. 1, pp. 4-15, January 1998.

[Côt98] G. Côté et al, "H.263+: Video Coding at Low Bit Rates", IEEE Transactions on Circuit and Systems for Video Technology, November 1998

[CSZ92] D. Clark, S. Schenker, and L. Zhang, "Supporting Real-Time Applications in An Integrate services packet network: Architecture and Mechanisms", Proc. SIGCOMM'92, 1992.

[DHT95] C. Diot, C. Huitema, T. Turletti, "Multimedia Applications should be Adaptive", Proc. HPCS'95, Mystic (CN), Aug. 23-25, 1995

[FRV92] D. Ferrari, J. Ramaekers, G. Ventra, "Client-Network Interaction in Quality of Service Communication Environments", $4^{\text {th }}$ IFIP Conference on High Performance Networking, University Liège, Belgium, 1992.

[F1J95] S. Floyd, V. Jacobson, "Link-sharing and Resource Management Models for Packet Networks", IEEE/ACM Transactions on Networking, Vol. 3 No. 4, pp. 365-386, August 1995.

[Gil60] E.N. Gilbert, "Capacity of a Burst-Noise Channel", The Bell System Technical Journal, September 1960.

[H261] ITU-T Recommendation H.261, "Video Codec For Audiovisual Systems and Terminal Equipment", March 1996.

[JDS95] S. Jamin, P. B. Danzig, S. J. Shenker, L. Zhang, "A Measurement based Admission control Algorithm for Integrated Services Packet Networks", Proc. SIGCOMM'95, 1995.

[MJV96] S. McCanne, V. Jacobson, M. Verletti, "Receiver-driven layered multicast", ACM Sigcomm'96, pages 117-130, 1996.

[MCC95] S. McCanne, V. Jacobson, "Vic: A Flexible Framework for Packet Video", Proc. ACM Multimedia'95, San Francisco, CA, November 1995.

[MeS99] J. Meggers, M. Schuba, "Analysis of Feedback Error Control Schemes for Block Based Video Communication", International Packet Video Workshop (PV' 99), New York City, April 1999

[RHE99] R. Rejaie, M. Handley, D. Estrin, "RAP: An End-to-end Rate-based Congestion Control Mechanism for Realtime Streams in the Internet", to appear in IEEE INFOCOMM 99.

[Sch96] H. Schulzrinne, “RTP A Transport Protocol for Real-Time Applications", RFC 1889, January 1996.

[Sis97] Dorgham Sisalem, "End-To-End Quality of Service Control Using Adaptive Applications", in IFIP Fifth International Workshop on Quality of Service (IWQOS '97), (New York, USA), IFIP WG 6.1, May 1997.

[Sis98] Dorgham Sisalem, "Fairness of Adaptive Multimedia Applications" in the proceedings of ICC'98, June, Atlanta 1998.

[SRC84] H. Saltzer, D.P. Reed, D. Clark, “End-to-end arguments in system design", ACM Transactions on Computing Systems, vol 2, no. 4, 1984. 
[TAL97] A. K. Talukdar, B. Badrinath, and A. Acharya, "On accommodating Mobile Hosts in an Integrated Service Packet Network”, IEEE INFOCOM' 97, April 1997.

[TSS97] David L. Tennenhouse, Jonathan M. Smith, W. David Sincoskie, David J. Wetherall, and Gary J. Minden, "A Survey of Active Network Research", IEEE Communications Magazine, Vol. 35, No. 1, pp 80-86. January 1997.

[TuH96] T. Turletti, C. Huitema, "RTP Payload Format for H.261 Video Streams", RFC 2032, October 1996.

[TUR96] T. Turletti, C. Huitema, "IVS Videoconferencing in the Net", IEEE ACM Transactions on Networking, Vol. 4, pp-340-351, June 1996.

[WiZ98] R. Wittmann, M. Zitterbart, “Amnet: Active Multicasting Network", Proc. of Intern. Conf. on Communications (ICC'98), Atlanta, GA, USA, June 1998. IEEE. 